\title{
Towards Autonomy, Self-Organisation and Learning in Holonic Manufacturing
}

\author{
Paulo Leitão ${ }^{1}$ and Francisco Restivo ${ }^{2}$ \\ ${ }^{1}$ Polytechnic Institute of Bragança, \\ Quinta Santa Apolónia, Apartado 134, P-5301-857 Bragança, Portugal, pleitao@ipb.pt \\ ${ }^{2}$ Faculty of Engineering, University of Porto \\ Rua Dr. Roberto Frias, P-4200-465 Porto, Portugal, fjr@fe.up.pt
}

\begin{abstract}
This paper intends to discuss self-organisation and learning capabilities in autonomous and cooperative holons that are part of a holonic manufacturing control system. These capabilities will support the dynamic adaptation of the manufacturing control to the manufacturing evolution and emergency, specially the agile reaction to unexpected disturbances.
\end{abstract}

\section{Introduction}

The manufacturing systems are complex non-linear systems, since the occurrence of a disturbance causes non-linear impact in the system. As some of the effects of the disturbance can remain in the system after the resolution of the event that originated the disturbance, their occurrence may have severe impact in the performance of manufacturing systems. For this reason, it is necessary to measure the system performance also in terms of response to change and capability to learn.

The unpredictability of disturbances and the need to keep the system at work independently of their size and rate puts new requirements to manufacturing control systems, which have to handle them dynamically and to tell the difference between an occasional disturbance and the evolution of the system environment. Additionally, the assessment of potential impact of identified disturbances assumes a crucial factor.

Holonic manufacturing concepts and multi-agent systems implementations seem to have the capability to answer to these new problems, due to their decentralisation, autonomy and cooperation features.

This paper describes a manufacturing control architecture, designated by ADACOR, based in holonic manufacturing concepts, that aims to address the dynamic adaptation of the manufacturing control to the manufacturing evolution, specially the agile reaction to unexpected disturbances, by introducing the concepts of autonomy, cooperation, learning and self-organisation in each holon and in whole system. 


\section{Holonic Manufacturing and Multi-Agent Systems}

The emergent requirements for distributed manufacturing systems, namely the dynamic adaptation to the manufacturing evolution and emergency, lead to the development of dynamic and adaptive manufacturing control systems based in new paradigms such as holonic manufacturing and multi-agent systems.

\subsection{Overview of Multi-Agent Systems and Holonic Manufacturing Systems}

The multi-agent system paradigm is characterised by decentralization and the parallel execution of activities based on autonomous entities, called agents. The definition of agent concept is neither unique nor consensual, existing some discussion in the scientific community about the issue, such as described in [1-3]. Despite the several definitions and interpretations for agents, a possible definition for agent is [4]:

An autonomous component, that represents physical or logical objects in the system, capable to act in order to achieve its goals, and being able to interact with other agents, when it doesn't possess knowledge and skills to reach alone its objectives.

Multi-agent system can be defined as a set of agents that represent the system objects, capable to interact between themselves, in order to achieve its individual goals.

The Holonic Manufacturing System (HMS) is a paradigm that translates to the manufacturing world the concepts developed by Koestler to living organisms and social organizations, mainly that complex systems are hierarchical systems formed by intermediate stable forms, being simultaneously a part and a whole [5]. The word holon is the representation of this hybrid nature, allowing that a holon can be part of another holon, e.g., a holon can be broken into several others holons, which in turn can be broken into further holons, which allows the reduction of the problem complexity. A holarchy is defined as a system of holons that can cooperate to achieve a goal or objective. A HMS is a holarchy, which integrates the entire range of manufacturing activities, where key elements, such as machines, products and robots, have autonomous and cooperative properties.

The agent-based and holonic manufacturing systems approaches are developed under the same principles of autonomy and cooperation, exploring the distribution and decentralisation of entities and functions. Although the similarity of the both concepts, there are some few differences that tend to be reduced, since at the moment the agent and holonic communities seem to be converging rapidly at the moment.

\subsection{Open Challenges}

In spite of the research developed using the holonic manufacturing paradigm, such as referred in [5-9], the holonic manufacturing paradigm presents some open questions on the design and implementation of manufacturing applications.

The first question is related to how it is achieved the global optimisation, since the holonic manufacturing approach is based in autonomous entities. In case of formation of hierarchies to achieve global optimisation, an open question is related to how temporary hierarchies are formed, managed and removed. 
In order to be able to integrate different holarchies, other open question is related with the definition of common ontologies to support inter-operability and knowledge sharing during the interaction processes. This inter-operability has two different levels: inter-operability within the same control platform and a more complex interoperability related to the integration of different (distributed) control platforms.

In order to adapt to disturbances, the implementation of self-organisation capabilities and the integration of planning, scheduling and plan execution functions, are yet far from trivial. The definition of how the learning capabilities of each holon should be improved to support the manufacturing evolution and emergency, also remains an open challenge.

At last, one drawback of holonic manufacturing paradigm is the few implementations using real industrial scenarios that can prove the advantages of the holonic approach.

\section{ADACOR Holonic Control Architecture}

The ADACOR architecture addresses the dynamic and agile adaptation to disturbances and the integration of all manufacturing functions, namely the process planning, the scheduling and the plan execution.

\subsection{Architecture Entities}

The architecture, based on the holonic manufacturing systems paradigm, is supported by a set of autonomous and intelligent holons, each holon being a representation of a manufacturing entity, such as a numerical control machine, a robot or an order. The ADACOR architecture considers four classes of manufacturing holons: the product, task, operational and supervisor holon [10]. Each product is represented by a product holon that contains all knowledge related to the product and process. Manufacturing orders to be executed in the factory plant are represented by task holons, which are responsible for the control and supervision of their execution. The operational holons represent the manufacturing resources, such as operators and robots, managing its behaviour and agenda according the resource goals, constraints and skills.

The product, task and operational holons are quite similar to the product, order and resource holons, presented at the PROSA reference architecture [5]. The supervision holon, not present in PROSA, introduces coordination and global optimisation in decentralised control approaches, coordinating several operational and supervisor holons. In normal operation, the supervisor holon supervises and regulates the activity of the holons under its domain, while when a disturbance occurs, these holons may have to find their way without the help of the supervisor holon. The supervisor holon is also responsible for the group formation, based in pre-defined clusters of holons, combining synergies, aggregating skills and offering the combined services to external entities in the manufacturing system. These groups can be formed to build a shop floor, a manufacturing cell, or a machine equipped with a set of tools, assuming the supervisor holon the control role of each group. 


\subsection{Fractal Holarchies}

ADACOR architecture presents self-similarity features, since each holon possesses a structure and behaviour similar to the ones of the holon where it is encapsulated. An operational holon can be a set of several operational or supervisor holons, allowing to build holarchies upon fractal holarchies. As an example, a manufacturing cell can be represented by an operational holon that comprises several other operational holons, each one representing a manufacturing resource, and one supervisor holon representing the manufacturing cell controller. In this case the supervisor holon acts as the logic component, and the several operational holons act as the physical part of the holon. Additionally, each one of those operational holons that represent a manufacturing resource can comprise several other operational holons, such as the numerical control machine itself and the several tools stored in its tool magazine.

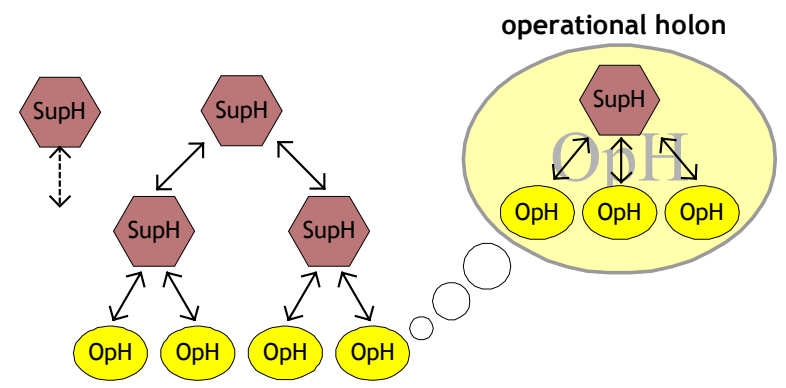

Fig. 1. Fractal Feature in ADACOR Approach

This fractal feature allows high flexibility in the organisation of the control structure, creating foundations to support the combination of global optimisation with agile reaction to disturbances. Additionally, it allows a modular development through the encapsulation of several functions or manufacturing components represented by holons in other holons.

\section{Self-Organisation Capability}

The industrial manufacturing systems are stochastic, volatile and dynamic environments. The heterarchical control architectures present good reaction to disturbances but degrade the global production optimisation; on the other hand, the hierarchical approach presents good global optimisation but weak reaction to disturbances. The challenge is to develop dynamic and adaptive manufacturing control approaches that improve the agility and reaction to unexpected disturbances without compromising the global optimisation.

The ADACOR architecture is neither completely decentralised nor hierarchical, but balances between one and the other [4], being the self-organisation crucial to reach the dynamic and adaptive control approach. 


\subsection{Autonomy Factor and Propagation Mechanisms}

The self-organisation capability presented in all ADACOR holons allows the dynamic adaptation of each holon and the system as a whole to the emergent contexts and the quick reaction to the occurrence of unexpected disturbances. This capability supports the re-organisation into different control architectures more appropriated to the current situation. Self-organisation is supported basically by the autonomy factor and the propagation of re-organisation concepts.

The autonomy factor, $\alpha$, associated to each operational holon is a parameter that introduces a fuzzy degree of autonomy in each holon, and evolves dynamically in order to adapt the holon behaviour according its goals and constraints and with the environment where it is placed. The autonomy factor is a continuous or discrete variable. Currently it is implemented using fuzzy logic, represented by the linguistic fuzzy sets $\{$ Low, High $\}$ and by a fuzzy-rule base. Normally, the operational holons have a $\{$ Low $\}$ autonomy factor, allowing the operational holon to follow the supervisor holon coordination [4]. The emergency triggers the change of the autonomy factor to $\{\mathrm{High}\}$, the re-organisation into a heterarchical control structure, and the selection of one adequate behaviour to handle the disturbance.

The need for re-organisation, using pheromone-like techniques, is disseminated to the supervisor holons through the propagation and deposit of pheromone to the neighbourhood supervisor holons [10]. While spreading the need for re-organisation, the holon passes a parameter that reflects the estimated reestablishment time $(\tau)$, similar to the odour from the pheromone-like techniques, and that is calculated according with the type of disturbance and with the historic data. The holons associated to each supervisor holon receive the need for re-organisation by sensing the pheromone, propagating this need to neighbourhood holons. The dissemination of the need for re-organisation, allows the dynamic and continuous adaptation of the system to disturbances, reducing the communication overhead and improving the reaction to disturbances.

Table 1. Fuzzy Rule Behaviour for the Adaptive Mechanism

\begin{tabular}{ccccc}
\hline$\rho$ & $\alpha$ & $\tau$ & New $\alpha$ & Action triggered \\
\hline High & Low & - & High & Trigger selection behaviour. \\
High & High & Elapsed & High & Reload reestablishment time. \\
Low & High & Elapsed & Low & Re-organise into default DF. \\
- & High & Not Elapsed & - & - \\
Low & Low & - & - & - \\
\hline
\end{tabular}

The autonomy factor, the reestablishment time and with the pheromone parameter $(\rho)$, that is concerned to the odour level of the pheromone or disturbance, jointly allow to regulate the adaptive behaviour of the operational holon. The adaptive mechanism determines the autonomy and the action to trigger according with Table 1.

In case that the action triggered be the selection of behaviour, it is necessary to know the disturbance type, the actual state of the holon and the historic data in order to select the appropriate behaviour and to estimate some required parameters. 


\subsection{Dynamic Production Control Structure Evolution}

The production control is shared between the supervisor holon and the operational holons, in order to balance the control structure from a more centralised approach to a more flat approach, passing through other intermediate forms of control [4].

The proposed adaptive control splits the control evolution into alternative states: stationary state, where the behaviour of the system uses coordination levels and the supervisor role to get global optimisation of the production process, and the transient state, triggered with the occurrence of disturbances and presenting a behaviour quite similar to the heterarchical approach in terms of agility to react to disturbances.

In stationary state the autonomy factor of each operational holon is $\{$ Low $\}$, allowing the operational holon to follow the schedule proposals sent by the supervisor holon. In this state, aiming the global production optimisation, the holons are organised in a federated architecture, with the supervisor holons interacting directly with the task holons during the operation allocation process. The supervisor holon, as coordinator, elaborates optimised schedule plans that proposes to the task holons and to the operational holons under its coordination domain [10]. The operational holons see these proposals as advices, having enough autonomy to accept or reject the proposed schedule. After the allocation of the manufacturing operations, the task holons interact directly with the operational holons during the execution of the operations, such as to ask for availability of space in the buffer.

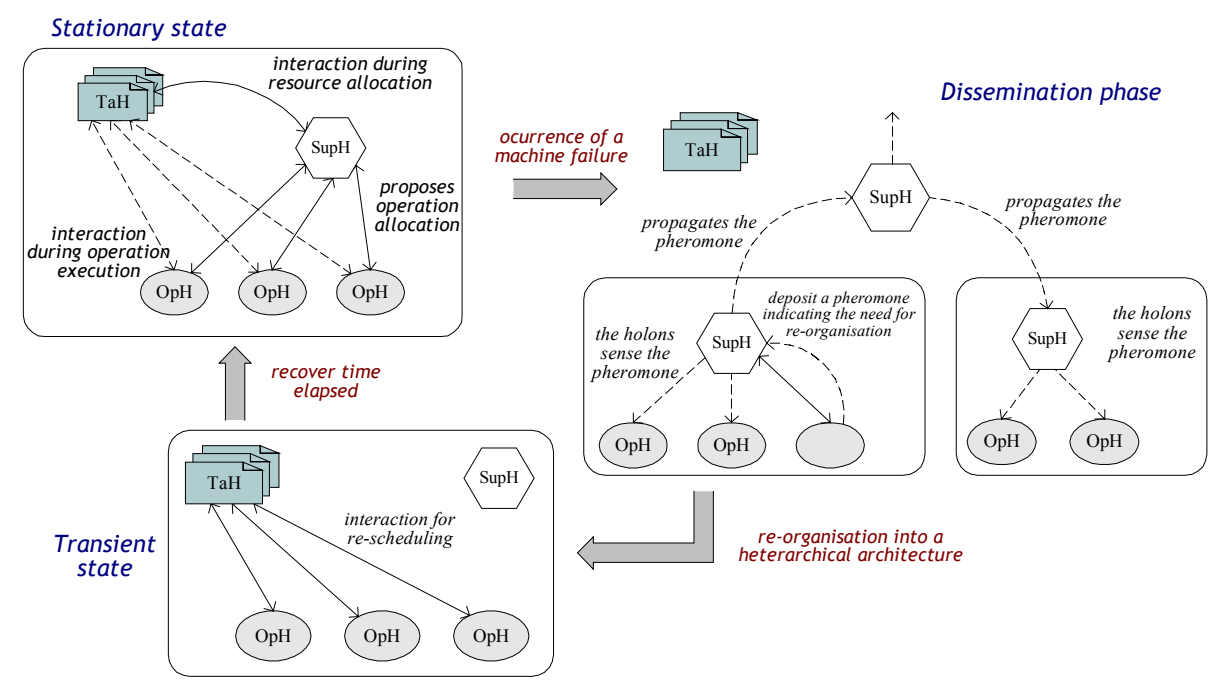

Fig. 2. Dynamic Re-organisation in Reaction to a Machine Failure

If, for any reason, the system deviates from planned, it is triggered a control system re-organisation, and it enters into the transient state. As illustrated in Fig. 2 for the case of a machine failure, the operational holon who detects the disturbance tries to recover locally the failure, but if it cannot recover from the failure, the operational holon increases the autonomy factor, disseminates the need for a re-organisation to the other holons in the system, and according with the type of disturbance selects an appropriate behaviour to handle the disturbance. 
The other holons that sense the propagation, also increase their autonomy factor and re-organise themselves into a heterarchical structure. In this state, the task holons can interact directly with operational holons to re-schedule their operations, achieving a faster but not optimised schedule plan. The operational holons remain in this transient state during the reestablishment period, typically a short period of time. When the time is elapsed, they verify if the odour is already dissipated or remains active. In case that the pheromone remains active, the operational holons stay in the transient phase during an additional proportional reestablishment time, until the pheromone be dissipated.

After the disturbance recovery, the operational holons end the reinforcement of the pheromone, and the reestablishment and recover times are adjusted and tuned using appropriated learning mechanisms. The other holons don't sense anymore the dissemination, reducing their autonomy factor, returning the system to the previous control structure. The supervisor holon returns to its coordination function, rescheduling the operations in an optimised point of view, and the optimised schedule to the operational holons.

\section{Learning Capability}

As an autonomous entity, an ADACOR holon is requested to take decisions about what actions it should perform. The introduction of learning capabilities in the holon behaviour intends to improve the holon and system performance.

\subsection{Learning to Support the Emergency and Evolution}

According to [12], learning in a multi-agent environment can help agents to improve their performance, namely to learn about the partner's knowledge and strategic behaviour, and to react to unexpected events by generalising what they have learned during a training stage.

Learning can be defined as a way to acquire knowledge and skills to adapt the behavioural tendencies, and it is crucial to respond to the dynamic evolution of the environment where it is placed and to improve the system ability to act in the future, by taking better decisions and performing better the required actions.

Learning is normally performed in result of a decision-making process allowing to adjust the decision parameters or even to update the behaviour rules. In the manufacturing control context, the learning mechanisms are triggered mainly in the following situations: when a process finishes (such as task allocation process and the end of the task holon life cycle), when the system configuration changes and when unexpected disturbances occur.

The ADACOR holons are self-optimised since they have the capability to continuously increase its performance using learning mechanisms. In spite of the wide range of learning techniques, such as described in [1,11-12], the proposed learning mechanism in ADACOR uses simple and reliable techniques. The mechanism stores the decision taken and uses the posterior results to learn. In the decision-making 
process, holons use the new knowledge acquired from previous experience to support the following decision-making, which will probably lead to better decisions and actions.

Depending of situation where learning is used, different learning techniques are applied, such as rote learning (simple memorisation) and unsupervised learning.

\subsection{Learning in the Prediction of Future Disturbances}

To exemplify the application of learning mechanisms in ADACOR holons, it will be described how to apply learning to predict future disturbances. As previously referred, the occurrence of disturbances degrades the system performance. The control system should be able to analyse the unexpected events and decide when an unexpected event is a real disturbance or a normal situation. The objective is to use appropriated learning mechanisms to find patterns in the occurrence of disturbances, foreseeing the stochastic effects of industrial environment, making predictable the occurrence of future disturbances, preparing the system to support these events.

The proposed approach uses an unsupervised learning mechanism based in the statistical clustering technique [11], to predict the time interval between consecutive disturbances. Cluster analysis aggregates objects (a vector of $n$ feature values) in clusters by analysing the similarity between them. A similarity metrics treats each object as a point in n-dimensional space, the similarity of two objects being the Euclidean distance between them in this space. The cluster formation is achieved such that the distance between any two instances in the cluster is less than the distance between any point in the cluster and any point not in it.

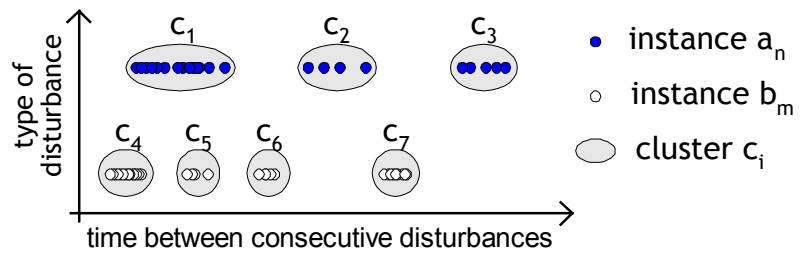

Fig. 3. Clustering of the Disturbances

The procedure involves, in a first step, the creation of groups of disturbances according to the similarities in the type of disturbances. In the next step each group is analysed individually, in order to find a pattern in the occurrence of that type of disturbance. In each group, obtained in previous step, the clustering algorithm described in [13] is applied, allowing to create clusters using the notion of a cluster diameter. This algorithm requires all data to be available prior to clustering, grouping the fault events in clusters, according to the similarities in the time between consecutive occurrences, as in Fig.3. In case that the distance between any two input pairs be the same, the location within the sorted list will be arbitrary and could lead to different classifications being produced.

After the detection of similarities between the historical disturbance events and the consequent creation of clusters, it is necessary to analyse all created clusters in order to detect the predominant cluster, which reflects the tendency of the disturbance 
sequence. A pertinent question is how to select the predominant cluster, since several parameters that characterize the disturbance sequence are not known in advance. The predominant cluster is selected taking in consideration the number of elements or the presence of the last disturbances. If the last disturbance events belong to the same cluster, that cluster reflects the tendency and it is considered predominant. Otherwise, the predominant cluster is the one whose contains the highest number of elements.

The last step is concerned to the analysis of the selected cluster and extraction of the predictability of occurrence of future disturbance, which based in estimation of the mean value of the cluster.

In order to validate the learning mechanism in the prediction of disturbances, its response was tested for several disturbance sequence inputs, as illustrated in Fig. 4.
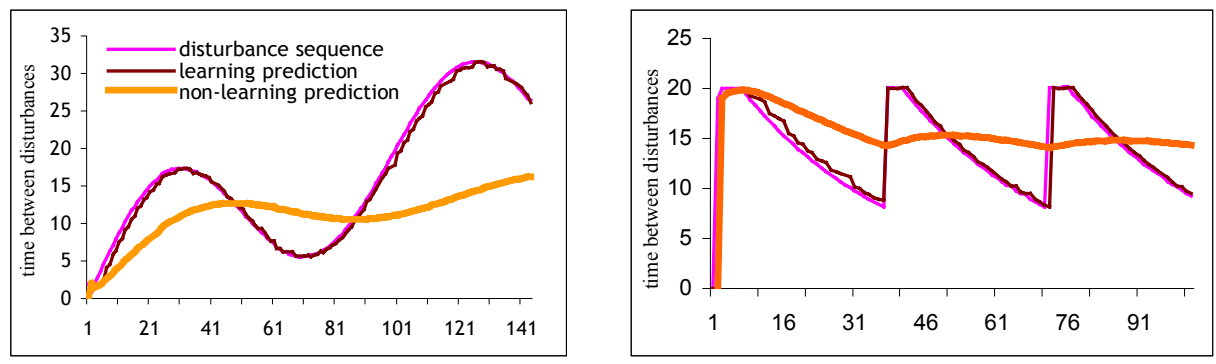

Fig. 4. Experimental Results

From the experimental analysis it is possible to verify that the proposed clustering mechanism presents a satisfactory precision in the prediction of the next disturbance, with precision over $95 \%$. Comparing with a particular non-learning technique, the one that predicts based in an average of past events, it is possible to verify the improvement achieved by using the learning mechanism in this situation.

In opposite to some techniques that require initial training, the clustering dispenses this training, achieving faster a prediction value. However, the clustering technique presents low performance in case of fewer fault events and is highly dependent of the cluster formation and selection. In order to improve the performance of the prediction mechanism, in further research work it will be considered not only the values of the time between the disturbances but also the feedback related to the precision about the prediction, applying for example a reinforcement learning technique.

\section{Conclusions}

The manufacturing systems are complex non-linear systems, since the occurrence of a disturbance causes non-linear impact and some effects of the disturbance can remain in the system after the resolution of the event that originated the disturbance. The development of manufacturing control systems that handle efficiently and quickly to the occurrence of disturbances is an actual and open challenge. 
The ADACOR architecture, based in holonic manufacturing paradigm, introduces the concepts of autonomy, cooperation, self-organisation and learning to support the agile reaction to unpredictable disturbances, such as machine failures and rush orders.

The self-organisation, based in the autonomy factor and propagation mechanism, allows to balance the control between different control structures, reaching an adaptive control approach that combines the agile reaction to disturbances with the global optimisation. The introduction of learning capabilities in manufacturing holons allows to improve the holon's ability to act in future and to support the dynamic evolution of the environment where it is placed.

As ADACOR is an open framework based on holons that can be built upon several building blocks (similar to Legos ${ }^{\circledR}$ components), further work should focus on the application of learning mechanisms for the identified situations using more powerful learning algorithms.

\section{References}

1. Russel, S., Norvig, P.: Artificial Intelligence, A Modern Approach. Prentice-Hall (1995).

2. Wooldridge, M., Jennings, N.: Intelligent Agents: Theory and Practice. In: The Knowledge Engineering Review, 10 (2), (1995) 115-152.

3. Ferber, J.: Multi-Agent Systems, An Introduction to Distributed Artificial Intelligence. Addison-Wesley (1999).

4. Leitão P., Restivo, F.: Holonic Adaptive Production Control Systems. In: Proceedings of $28^{\text {th }}$ Annual Conference of the IEEE Industrial Electronics Society, Spain, (2002) 2968-2973.

5. Van Brussel, H., Wyns, J., Valckenaers, P., Bongaerts, L., Peeters, P.: Reference Architecture for Holonic Manufacturing Systems: PROSA. In: Computers In Industry, 37 (3), (1998) 255-274.

6. Christensen, J.: Holonic Manufacturing Systems - Initial Architecture and Standard Directions. In: Proceedings of the First European Conference on Holonic Manufacturing Systems, Hannover, Germany, (1994).

7. Fisher, K.: Agent-Based Design of Holonic Manufacturing Systems. In: Journal of Robotics and Autonomous Systems, Elsevier Science B.V., 27, (1999) 3-13.

8. Bussman, S.: An Agent-Oriented Architecture for Holonic Manufacturing Control. In: Proceedings of $1^{\text {st }}$ Workshop on the Esprit Working Group on IMS, Lausanne, (1998) 1-12.

9. Brennan, R., Fletcher, M., Norrie, D.: An Agent-based Approach to Reconfiguration f RealTime Distributed Control Systems. In: IEEE Transactions on Robotics and Automation, 18(4), (2002) 444-451.

10. Leitão, P., Restivo, F.: A Holonic Control Approach for Distributed Manufacturing. In: Marik, V., Camarinha-Matos, L., Afsarmanesh, H. (eds.): Knowledge and Technology Integration in Production and Services: Balancing Knowledge and Technology in Product and Service Life Cycle. Kluwer Academic Press, (2002) 263-270.

11. Kubat, M., Bratko, I., Michalski, R.: A Review of Machine Learning Methods. In: Michalski, R., Bratko, I., Kubat, M. (eds.): Machine Learning and Data Mining: Methods and Applications. John Wiley \& Sons, (1996) 1-72.

12. Goldman, C.V. and Rosenschein, J.S: Mutually Supervised Learning in Multiagent Systems. In: Weiss, G. and Sen, S. (eds.): Adaptation in Multi-Agent Systems. Lecture Notes in Artificial Intelligence, Springer-Verlag, (1996) 85-96.

13. Hutchinson, A.: Algorithmic learning. Clarendon Press, (1994). 